\section{A) Check for updates}

Cite this: Nanoscale, 2020, 12, 21409

\title{
Structural colouration in the Himalayan monal, hydrophobicity and refractive index modulated sensing $\dagger$
}

\author{
ljaz Rashid, (D) a Muhammad Umair Hassan, (D) a Muhammad Nazim, ${ }^{a}$ \\ Mohamed Elsherif, ${ }^{b}$ Qian Dou, ${ }^{c}$ Debo Hu, (D) ${ }^{c}$ Muhammad Kamran, ${ }^{a}$ Qing Dai (D) \\ and Haider Butt (D) *a,b
}

\begin{abstract}
The Himalayan monal is a bird in the pheasant family, and it is the national bird of Nepal. The bird possesses spectacular iridescent plumage with a range of different metallic colours. Here, we have studied the internal structure of its feathers from different parts of the bird's body and showed that its beautiful colours and iridescence are due to photonic structures present in the internal structure of the feathers. Sharp changes in the reflected brilliance were observed from the feathers upon changing the illumination conditions, such as horizontal and azimuthal angles. The feathers exhibited interesting hydrophobic properties, with the dull-coloured proximal end showing lower hydrophobicity with a contact angle between $90^{\circ}$ and $110^{\circ}$ compared with the iridescent distal end of a feather exhibiting a contact angle between $115^{\circ}$ and $120^{\circ}$, attributed to the change in the internal structure and/or density of the feathers. A quick reversible change in colours of these feathers was observed when they were soaked in water and other liquids, which reversed upon drying. The shift in colour was suggested to be due to the swelling of the keratin layer of barbules that absorbed liquids and as a result modified the refractive index and periodicity of the internal photonic structures. The colour shift response of feathers was different in the case of alcohols and other water-based solutions, suggesting different swelling behaviour of keratin against different liquids; the water-based solution had the more pronounced effect. Such photonic modulation can be utilized in colour selective filters and sensing devices.
\end{abstract}

Received 5th September 2020, Accepted 23rd September 2020

DOI: $10.1039 /$ d0nr06382g

rsc.li/nanoscale action gratings, and photonic crystals, and also due to other physical parameters, such as size and refractive index. ${ }^{11}$ Such colouration is useful for noticeable signals intended for intraspecific communication, sign of warning or flash and startle displays. ${ }^{12}$ The viewing angle significantly varies the hue and/or intensity of iridescent colours which allows animals and insects to be noticeable to the intended signal receiver, whilst remaining reasonably discrete to avoid conflict with nearby predators. ${ }^{12}$

Photonic structures are seen in many avian taxa and vary within groups of birds that differ in size, behaviour, and ecology. ${ }^{13}$ The essential characteristic of a photonic crystal is the periodicity of the dielectric material along one or more axes, ${ }^{14,15}$ hence they can be one, two or three-dimensional, which is also the basis for their basic division. The photonic structure of a barbule is mainly derived from melanosomes, keratin and air and can be arrayed in one-dimensional laminar nanostructures, two-dimensional crystal nanostructures, which may have square or hexagonal lattices, or even three-dimensional nanostructures. ${ }^{16}$ Iridescent feathers of domestic pigeons were found to be composed of a surface keratin layer surrounding a medullary layer and it was discovered that the 
structural colour originates from the light interference in this keratin surface layer, ${ }^{17}$ classed as one-dimensional in the form of multilayers. A study of wing patches of dabbling ducks showed that the iridescent colours of the duck specula are formed by two-dimensional hexagonal photonic crystals (PCs) of rod shaped melanosomes set beneath a thin layer of keratin at the surface of the feather barbules. ${ }^{18}$ Two-dimensional PCs have also previously been expressed in peacocks, ${ }^{7}$ which also belong to the pheasant family. These are unlike those observed in ducks because their lattices are square rather than hexagonal and have air between melanosomes rather than keratin. ${ }^{18}$ The intensely coloured iridescent feathers of several hummingbird species, for instance, have been discovered to be because of the coherent scattering of light from multiple, interchanging layers of keratin and hollow air-filled, disc-shaped melanin granules. ${ }^{19,20}$ The iridescent colour of the male Peafowl (Pavo cristatus), by contrast, is created by crystal-like arrays of solid, rod-shaped melanin granules. ${ }^{16}$ These different types of structures show that a wide range of possibilities of PCs can be arranged in nature.

The aim of this study is to investigate the structural properties and optical effects of the feather barbules of the male Himalayan monal (Lophophorus impejanus, Fig. 1). The bird's feathers possess a layered internal structure as determined by transmission electron microscopy (TEM) that causes its brilliant metallic colours. The effect of different liquids on the colours of the bird's feathers was studied. The feather behaved differently to alcohol and water based solutions. Modulation of photonic properties of these feathers can be utilized in colour selective filters ${ }^{21-23}$ and colorimetric sensors. ${ }^{24-26}$ The large wavelength shift as well as the angle selectivity in these kinds of structures can potentially offer high sensitivity and large turn-on-off ratios. ${ }^{27}$

\section{Results and discussion}

To provide a macroscopic view of the effect of the varying observation angles on the feathers' perceived colour, a camera was used to take pictures at 0 and $45^{\circ}$ angles of incidence under a normal ambient illumination (Fig. 2a). The pictures taken for the red, green and blue feathers showed distinct shifts in the intensity of their respective colours between iridescent metallic and dark when the illumination angle changed between 0 and $45^{\circ}$, respectively. Feathers' barbules were vivid in colour and had metallic perception, however, the downy barbs and after feathers appeared dark grey, and did not exhibit any visible colouration (Fig. $2 \mathrm{c}$ and d, photographic images).

Optical spectra were also recorded at normal to the feather surface with an illumination angle of $\sim 15^{\circ}$, whilst the feather was rotated through a rotational stage with increments of $10^{\circ}$ between 0 and $360^{\circ}$ (Fig. 2e). The experiment was conducted to appreciate the drastic appearance and disappearance of the reflected beam in terms of the spectral intensity - to an observer, the feather appears bright metallic at some angle and

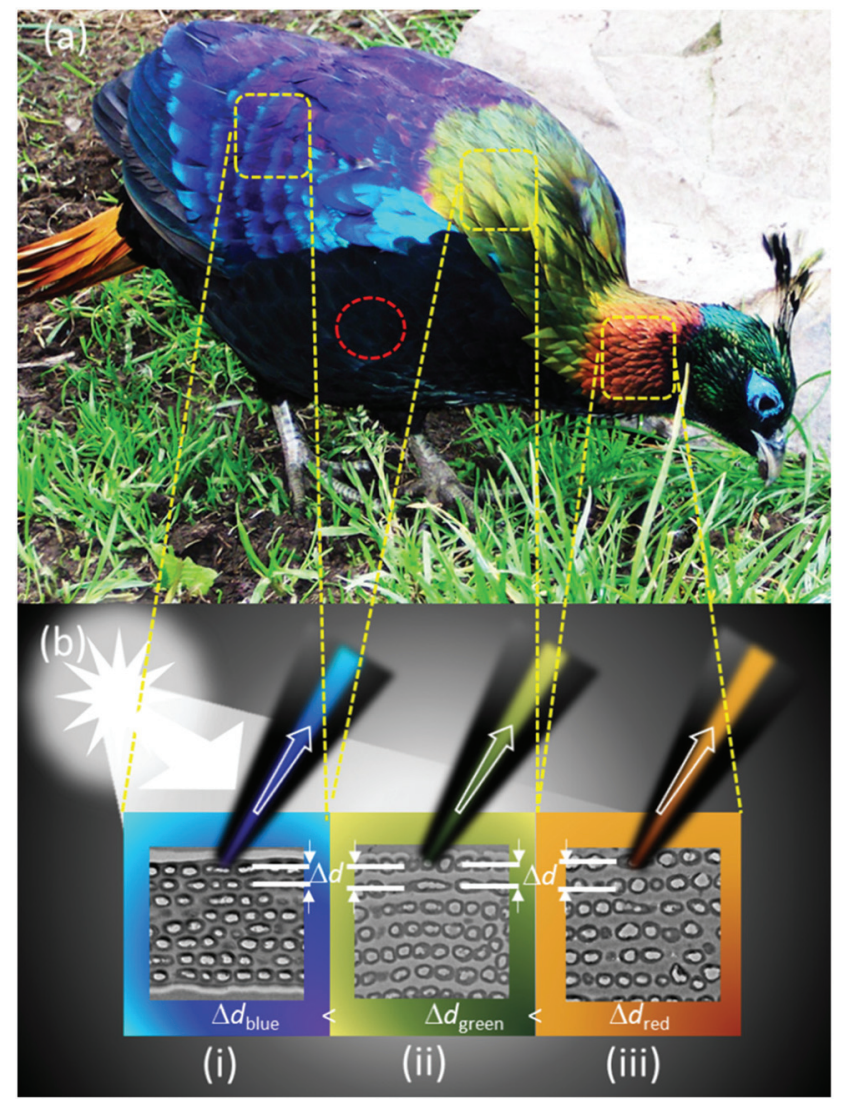

Fig. 1 Himalayan monal (Lophophorus impejanus). (a) The bird is a pheasant and the national bird of Nepal. Its strong and beautiful angle dependent iridescence (see yellow and red contours) is due to the underlying photonic structures in its plumage. The range of colours, from red to blue, is due to the size effect of the photonic structure. (b) Illustration of the selective Bragg's reflection by showing the internal structure of the feathers taken from different parts of the bird's body.

becomes very dark when there is a slight change in the viewing angle. Measurements were taken on the most iridescent spots (diameter $=4 \mathrm{~mm}$ ) on the feathers (Fig. $2 \mathrm{f}-\mathrm{h}$ ). To observe the angle dependence of the reflected light intensity, peak maxima obtained from the reflection spectra were also plotted against the rotation angle. The red feather showed the strongest change in intensity upon rotation through $360^{\circ}$. The feather exhibited two intense peaks (angular span of $\sim 80^{\circ}$ ) during the complete rotation, beyond which it appeared very dark. The green feather showed a broader angular span of $150^{\circ}$ of high reflection intensity having two overlapping peaks, and the feather appeared dark in the remaining angles. The blue feather showed multiple peaks through $360^{\circ}$; however, it never became completely dark (giving intensities below the lower resolvable limit) during the full $360^{\circ}$ rotation. It was noticed that the arrangement of barbules in feathers at the macroscopic scale severely affected the overall reflection behaviour. Feathers at the bird's back had a slightly different arrangement compared to the feathers at the neck and tail. By the same token, the location on the feather on which measure- 

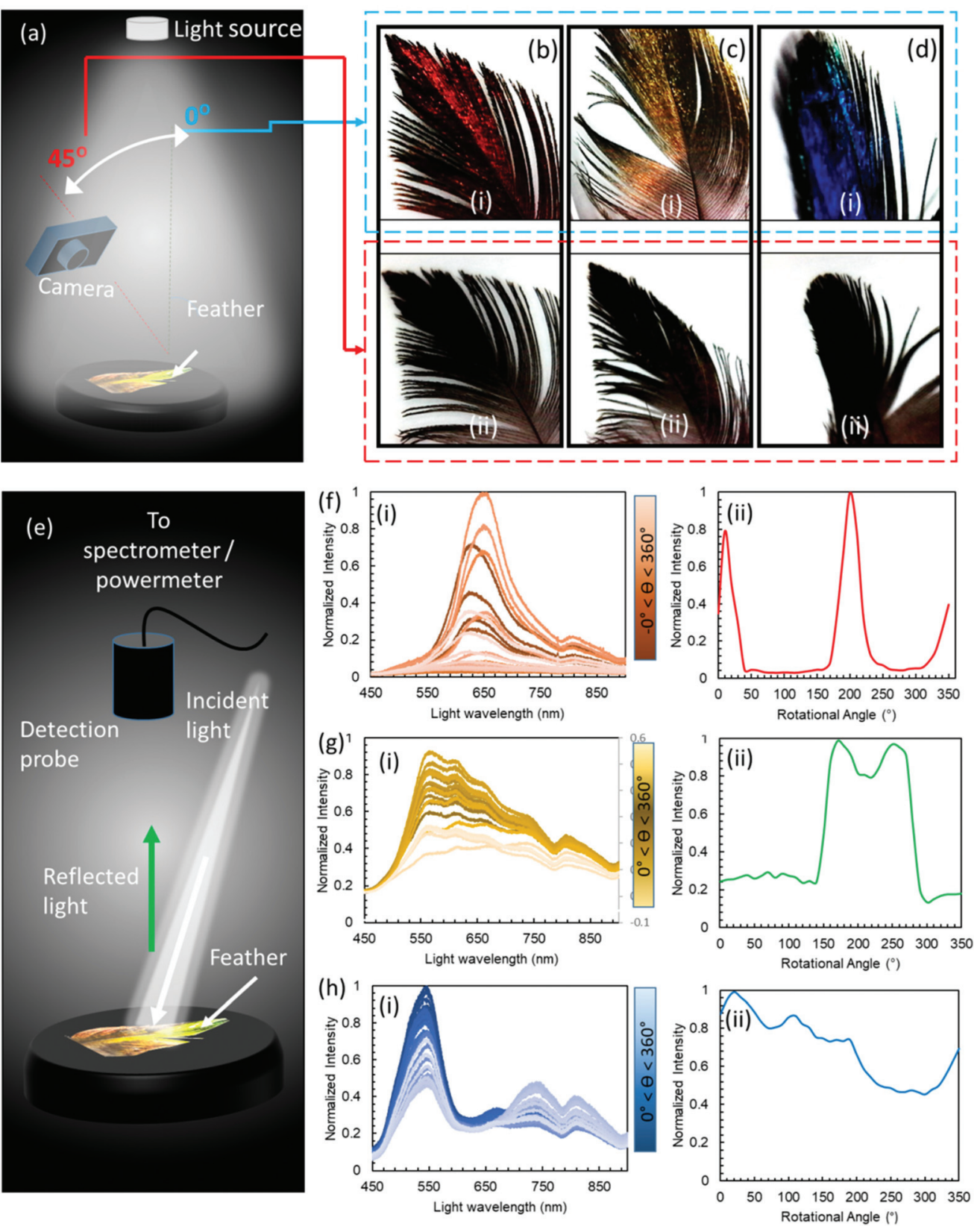

Fig. 2 Colour perception of the Himalayan monal's plumage. (a) Schematic for different observer angles in ambient light. (b-d) Images of red, green and blue feathers at an angle of observation at (i) $0^{\circ}$ and, (ii) $45^{\circ}$, respectively.(e) Schematic for a normal observation angle with illumination at an angle of $15^{\circ}$, and, feather rotation through $0-360^{\circ}$. ( $\mathrm{f}-\mathrm{h}$ ) Behaviour of the reflected beam with the changing rotation angle of red, green and blue feathers: (i) reflection spectra with varying rotation angles and (ii) peak intensities with variation in rotation angles, respectively.

ments were taken also affected the angle dependence of the reflected light, that is, feathers have different arrangements and densities close to the main shaft compared to the distal ends. Nevertheless, all iridescent parts of these feathers showed high angular dependence on the reflected intensity. Structural colours show angular dependence by appearing iridescent/metallic at certain angles and dark on others. ${ }^{28}$ The internal nanoscopic structures define the characteristics of a structural iridescent feather. The observed angular dependence of a monal's feathers suggested that 2D photonic crys- tals (multi-layered structure) could be present in these feathers, similar to some other avian species. ${ }^{29,30}$

Dark and bright field microscopy modes (Ziess, 20×) were used to observe the surface, exterior's structure and colours of red, green and blue feathers of the Himalayan monal under two illumination settings (Fig. 3d-f). In the bright field (BF) mode, each feather's iridescent barbules appeared enlarged, elongated, flattened and twisted at the base so that the flattened surface of the barbules came parallel with the surface of the feathers. The flattening barbules stacked up on each other, 

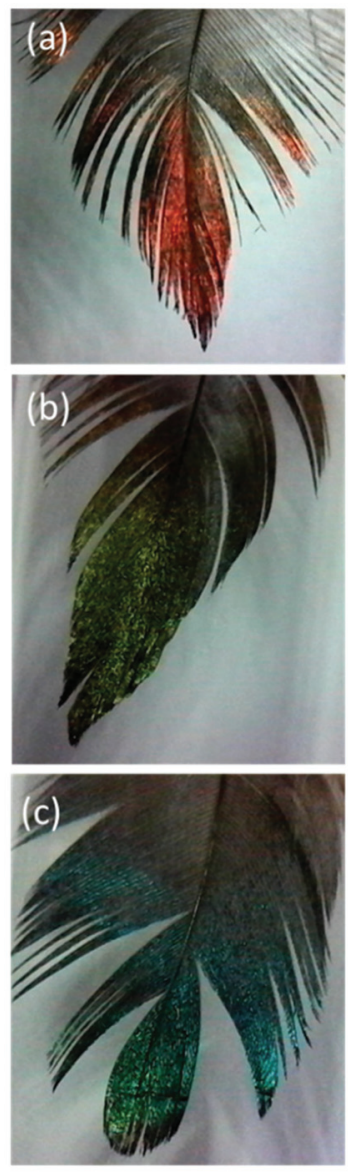
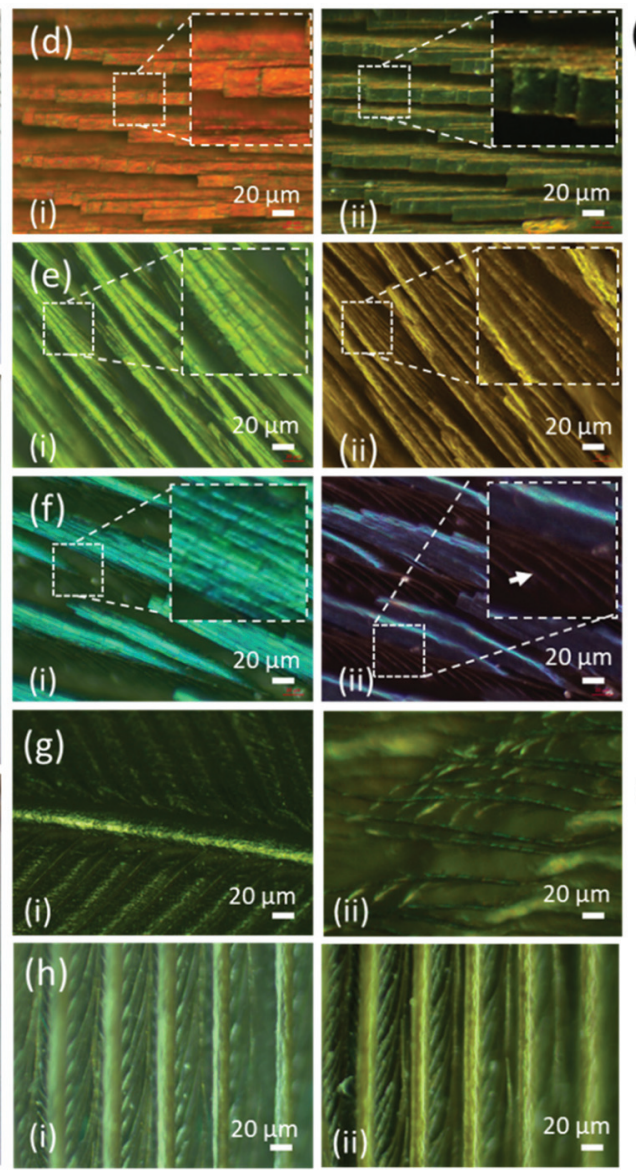
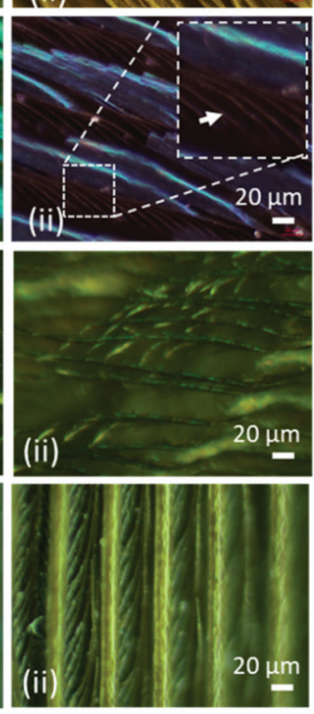

(i)
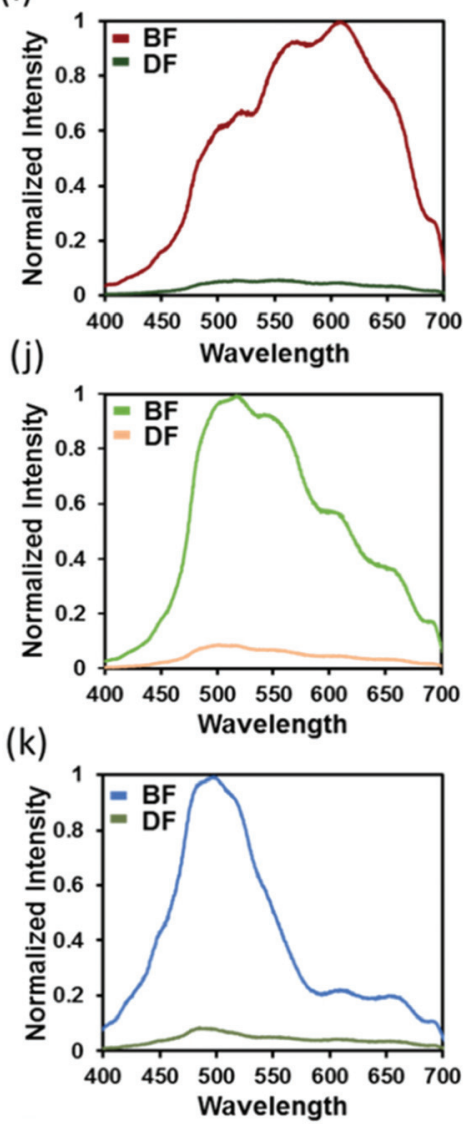

Fig. 3 Feathers of the Himalayan monal and their colours. $(a-c)$ Photographic images of red, green and blue feathers, respectively. (d-f) Brightfield (i) and dark-field (ii) microscopic images of the red, green and blue feathers taken from the top iridescent to side of the feathers. (g) Bright field (i) and dark field (ii) microscopic images of the underside of the blue feather, all other feathers (not shown here) appeared the same from the bottom. (h) Bright field (i) and dark field (ii) images of the proximal region showing the lack of colour in this particular region of the feather. Scale bar: $20 \mu \mathrm{m}$ unless stated otherwise. (i-k) Visible reflected spectra recorded in the bright and dark field modes: strong reflection behaviour was observed in the bright field mode, while the feathers in the dark field mode were almost completely non-reflecting.

orienting several reflecting parallel structures at the same angle. The distinct barbule morphology is prevalent amongst birds with iridescent colour that improves the effectiveness of iridescent colour displays. ${ }^{16,28}$ The increased number of stacked reflecting planes (barbules) in the Himalayan monal increased their vividness and made them appear extremely metallic. When compared to the less coloured proximal end of the feather (Fig. 3g), the negative impact that the reduced available surface area/planes had on its ability to reflect incident light was significant. Another important feature of these feathers was their geometric truncation of barbules. The barbule ends had sharp rectangular-faced terminations, which may be due to their internal highly ordered structure/photonic crystals. The under feather appeared dark grey and did not exhibit any visible colouration (Fig. 3h). Microscopic images of all feathers taken in the BF mode showed red, green and blue colours, while dark field (DF) images appeared dull as if no reflection was reaching to the eyepiece, indicating the angle selectivity of such photonic colouration. Notice that, in the BF, the light is incident from the top, while the imaging is also carried out from the same angle, whereas, in the DF, the illumination occurs at a certain angle and scattered light is collected from the object placed with the plane normal to that of the object (feather). The observation is consistent with that generally experienced at the macroscopic scale. It was observed that barbules and hooklets attached with barbs (in the vane section) were responsible for iridescent colours and photonic effects in all feathers. Barbs, rachis, or shaft did not exhibit photonic effects.

The camera at the eyepiece was replaced with a spectrometer (Ocean Optics, USB2000+) and reflection spectra were recorded from the same locations of red, green and blue feathers in the BF and DF modes (Fig. 3i-k). All feathers showed a clear change in the intensity of the colour/wavelength peak, with the barbules appearing bright (high intensity) in BF mode, whereas they appeared dark (low intensity) in the DF reflecting mode - no appreciable change in the peak wavelengths was observed. Normalized wavelength intensity graphs 
were plotted and the peak wavelengths for the red, green and blue feathers were found to appear at 2620,520 , and $495 \mathrm{~nm}$, respectively. The reflection of the underside of the feather was not within the resolvable limit of the spectrometer, while observed in both BF and DF modes. Since all feathers showed a large change in the intensity of the colour with changing illumination angle, it can be deduced that the observed colours were due to the internal structure of the feathers. ${ }^{31,32}$ Such colouration behaviour (selective wavelength reflection and angular dependence) is the characteristic feature of photonic crystals.

TEM images were obtained to see the details of the internal structure of the red, green and blue feathers (Fig. $4 \mathrm{a}-\mathrm{c}$ ). It was determined that the feather barbules of the Himalayan monal contain hollow melanin granules, running parallel to the barbule surface, arrayed in 6-9 layers and perhaps connected by keratin. Oval-shaped hollow melanosome-like structures were observed that were aligned in the form of layers as in a Bragg reflector. To define the class, the shape of melanin granules of the Himalayan monal was categorized as R-type (hollow tube), and the arrangement of granules was S-type (multilayer), considering Durrer's classification. ${ }^{15,30}$ Therefore, the generic structure among all feathers was considered to be the RS-type. A close look revealed that the size of hollow melanin granules did not change significantly for all feathers. The average radii along the minor axes of these structures were noticed to be almost the same, that is, $300( \pm 30) \mathrm{nm}$ for red green and blue feathers, respectively. However, it was noticed that the distance between their layers changed significantly - the average distances between the layers were $\sim 147( \pm 20), 110$ and $63 \mathrm{~nm}$ for
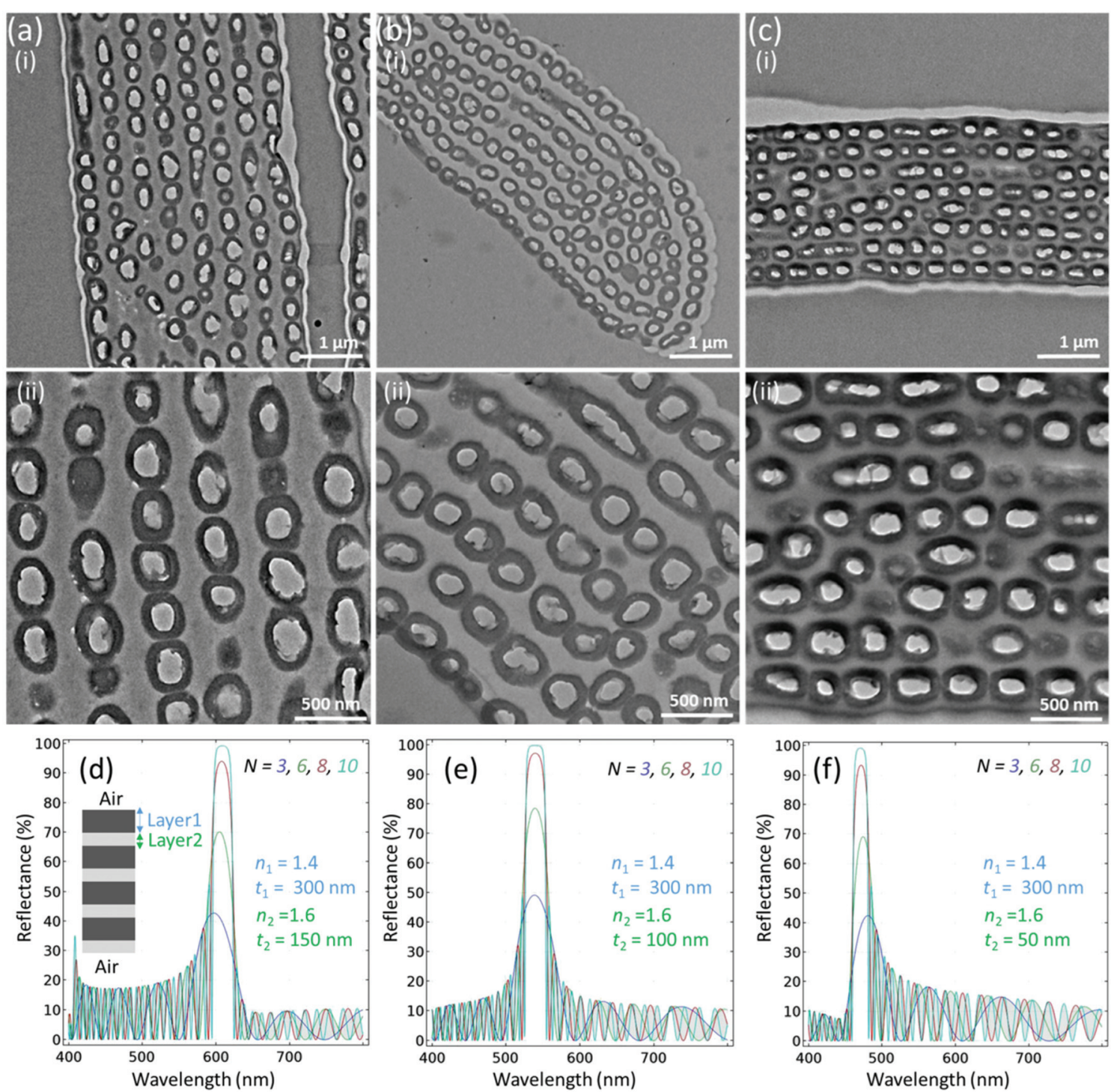

Fig. 4 Internal structure of the feather. (a-c) TEM images of red, green and blue feathers respectively with (i) low and (ii) high zoom. All feathers had a layered internal structure. ( $d-f$ ) Finite element simulation of layered structures with different periodicities shows different reflections comparable to red, green and blue feathers, respectively. 
red, green and blue feathers, respectively. Keratin and melanin have been reported to have various refractive indexes causing light to be reflected at the boundaries (and at the interface with air) and thus the barbules act as optical multilayers, similar to other reports. ${ }^{29}$ It has been observed that the contrast of the refractive index between the outer and inner melanin layers could be quite significant, $n_{\text {outer }} \approx 2$ and $n_{\text {inner }}$ $\approx 1$, thus the constructional interference that occurs is very noticeable in the form of colour change. ${ }^{29}$ A continuous layer of keratin generally overlies the melanin granules. The refractive index of the surface keratin layer and the change in refractive index with the surrounding medium air $(n \approx 1)$ also produce the structural colour, as the overall barbule refractive index depends on the relative amounts of keratin and melanin in the feather's body. ${ }^{33}$

We used the finite element method to model the estimated emission profile of the layered structure (Fig. 4d-f). For reflectance, we used the following equation

$$
R=\left[\frac{1-\left(n_{1} / n_{2}\right)^{2 N}\left(n_{1} / n_{\mathrm{air}}\right)}{1+\left(n_{1} / n_{2}\right)^{2 N}\left(n_{1} / n_{\mathrm{air}}\right)}\right]^{2}
$$

where $n_{\text {air }}(=1)$ is the refractive index of air, $n_{1}(=1.4)$ and $n_{2}$ $(=1.6)$ are the refractive indices of the alternating layers, while $N$ is the number of layers involved in the structural reflection process. For the sake of simplicity, multiple layers of continu- ous films were used. ${ }^{17}$ The peak intensities for the three different sets of parameters were observed at $\sim 610,540$, and $460 \mathrm{~nm}$, which is in agreement with that observed for different feathers under discussion.

Wettability of a solid surface is typically measured by the contact angle (CA) that water makes with it. As is well known, the CA is defined as the angle between the solid surface and the tangent to the liquid surface (on the liquid side of it), at the three-phase contact line. It is customary to define a surface as hydrophilic if $\mathrm{CA}<90^{\circ}$, and hydrophobic otherwise. Superhydrophobic and self-cleaning properties of the surface usually are derived from a very high advancing water contact angle, typically of $150^{\circ}$ or higher, and a very low contact angle hysteresis, which is a very low sliding angle, typically below $10^{\circ} .^{34,35}$ The wetting properties of feathers were tested by placing a water droplet $(2 \mu \mathrm{l})$ on each of the two main areas of the feather, the proximal and distal ends (Fig. 5d). The results suggested that each end had slightly different wettability. The overall feather surface was hydrophobic as all recorded contact angles were over $90^{\circ}$. The droplet had a contact angle of $\sim 120^{\circ}$ at 0 minutes in the less coloured, proximal area of the feather, and changed to $\sim 110^{\circ}$ after 5 minutes. The droplet on the more coloured, distal area of the feather exhibited a smaller contact angle of $\sim 110^{\circ}$, and that after 5 minutes, reduced to $\sim 90^{\circ}$. Hydrophobicity in feathers is mainly attributable to the width and spacing of barbs and barbules, arrayed in a way that

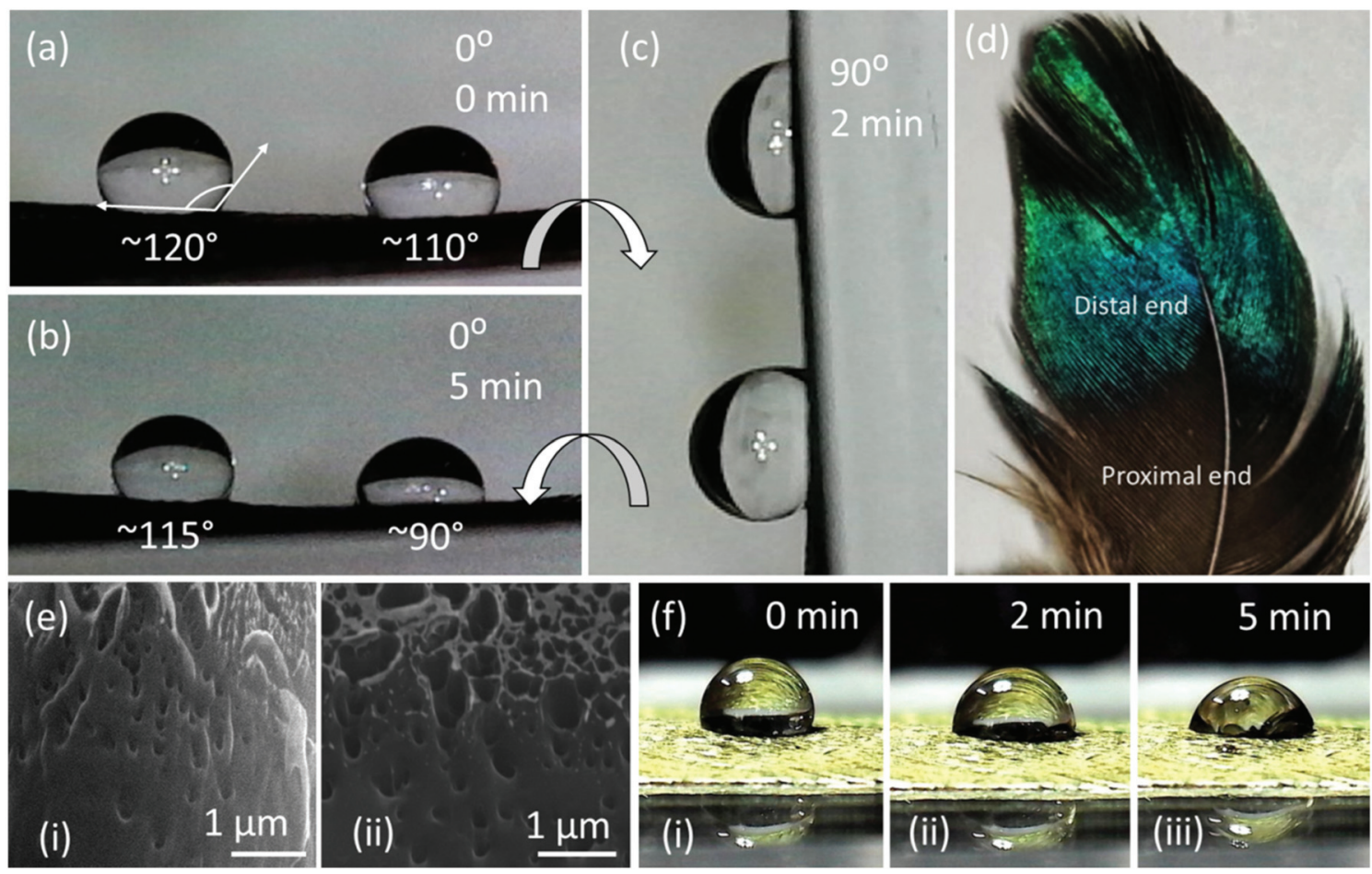

Fig. 5 Hydrophobicity and SEM images of the feather samples. (a) Image of the contact angles of two $2 \mu \mathrm{l}$ water droplets at $0^{\circ}$ tilt angle, one in the proximal area of the feather and one in the distal area. (b) Image taken 2 minutes later, with a tilt angle of $90^{\circ}$. (c) Image taken 5 minutes later, with a tilt angle of $0^{\circ}$ (d) Feather with distal and proximal ends marked, for clarity. (e) SEM micrographs of the feather barbule. (i) and (ii) represent the SEM images of proximal and distal ends respectively. (f) Images of change in contact angle on the distal end showing absorption with the time lapse. 
reduces contact between water and hydrophilic keratin. ${ }^{36}$ The decreased hydrophobicity of the feather could suggest a tradeoff between the iridescence and hydrophobicity in these feathers. Droplets poured on the distal and proximal parts of the feather did not fall off and remained attached to the surface when the feather was tilted at $90^{\circ}$, which is attributed to the large hysteresis range of the thermodynamic contact angles as most of the droplet base is in contact with the feather's rough surface rather than with air. Scanning electron microscopy (SEM) of Au-coated feather revealed pores on the surface of the barbule that can also result in increased water affinity on these feathers. The hydrophobic surface of feathers is useful for birds, because the water droplets rolling off the surface carry any surface contaminants with them. ${ }^{37,38}$ The experimental analysis and interpretation presented here is specific for the sample feathers tested under laboratory conditions. The characteristics of these feathers on a living bird may differ and depend on the climatic conditions of the natural habitat.

\section{Water sensitivity}

Interesting reversible colour shifts were recorded when the feathers were soaked in water (Fig. 6). Photographs, microscopic images and spectral data were obtained to observe the difference between wet and dry states. The soaking allowed the feathers to absorb water, which resulted in a colour shift. The red feather showed a strong blue-shift (656 to $496 \mathrm{~nm}$ ) and turned blue on soaking in water (Fig. 6ai-vi and c), whereas green and blue feathers exhibited red-shifts from 548 to $644 \mathrm{~nm}$ (Fig. 6bi-vi and d) and 495 to $500 \mathrm{~nm}$ (Fig. 6ci-vi and f), respectively after they were soaked in water. As a general
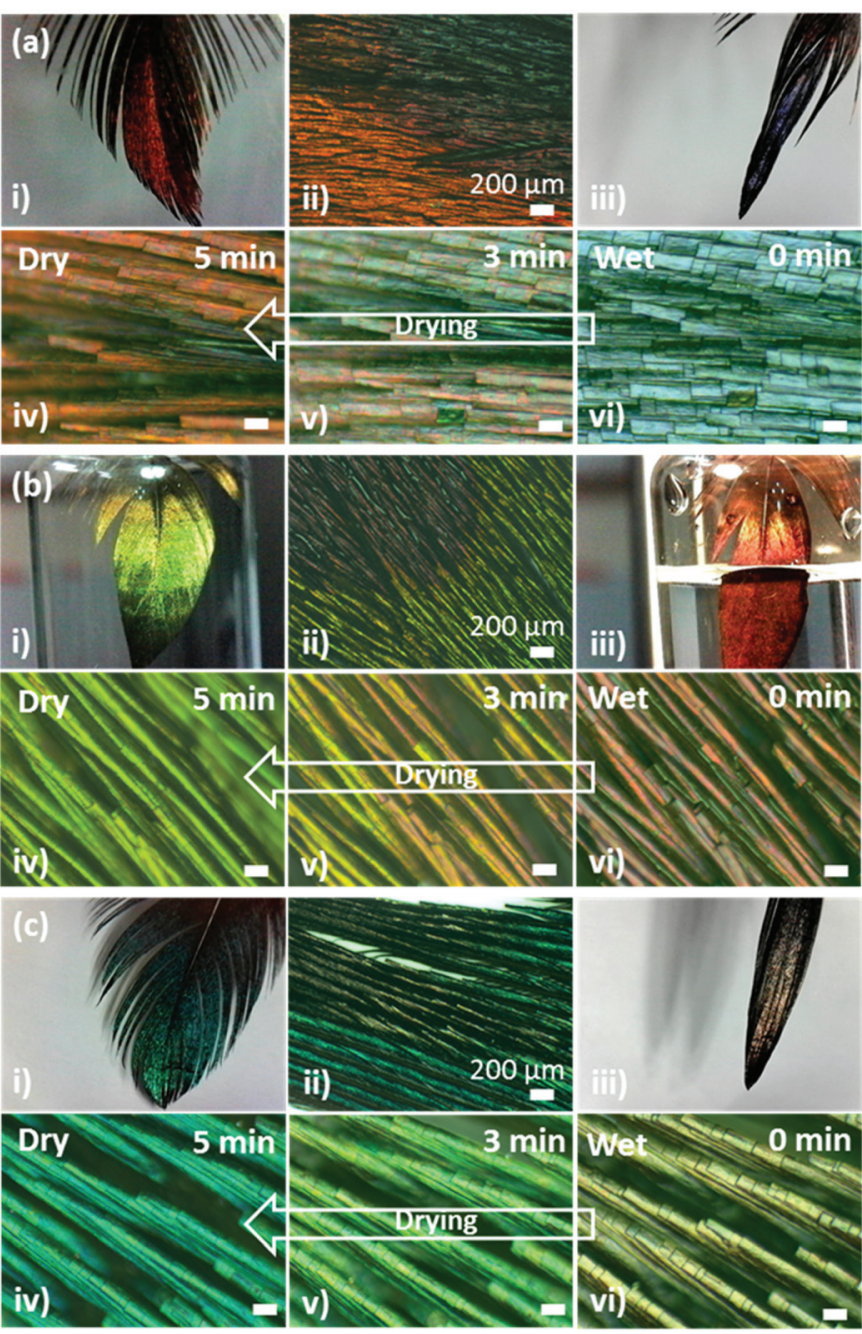
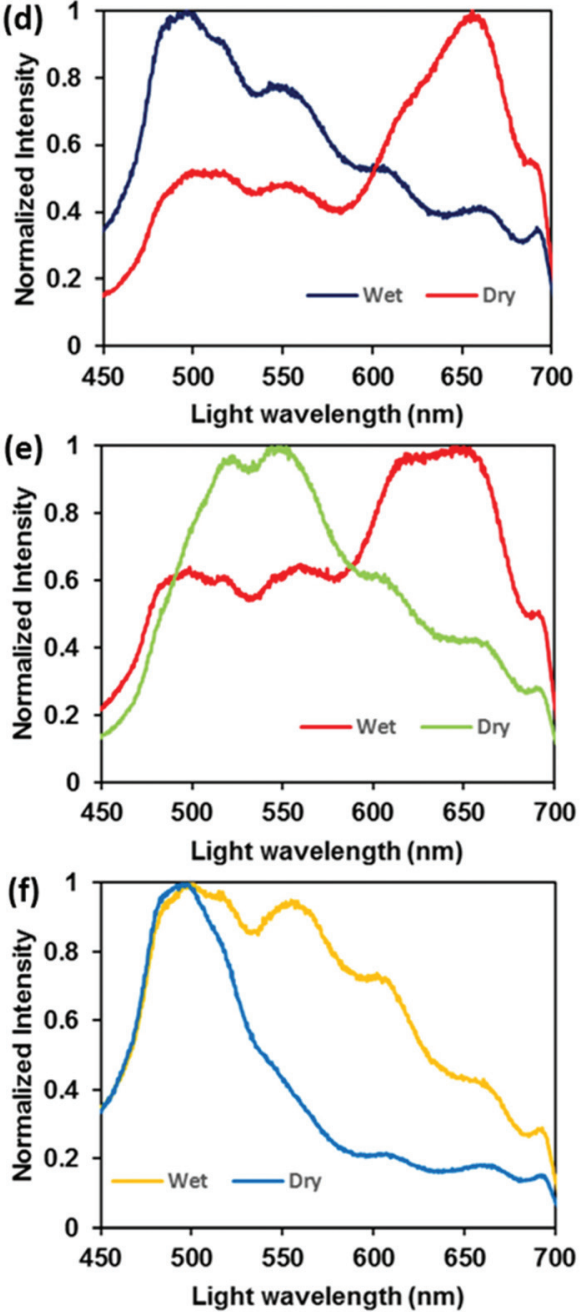

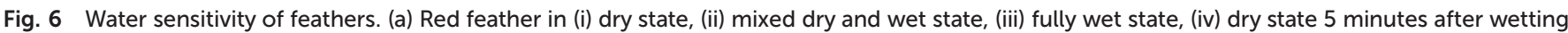

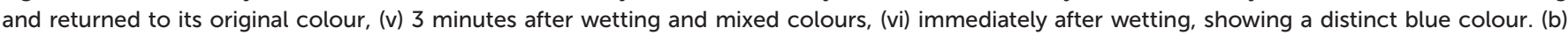

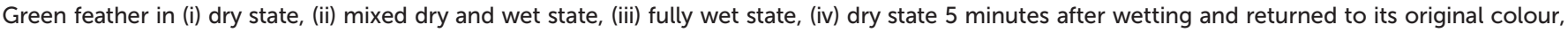

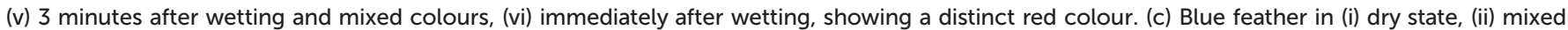

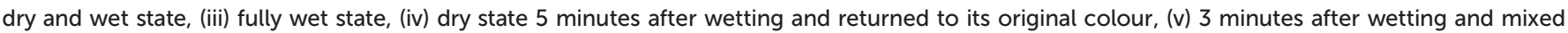

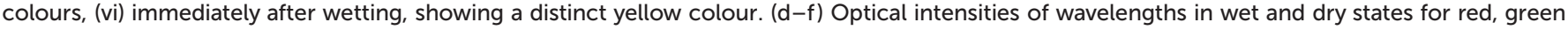
and blue feathers respectively. Scale bar: $20 \mu \mathrm{m}$. 
trend, the overall spectra broadened significantly under the soaked condition. The change in colour in the wet state occurred within $30 \mathrm{~s}$ and had reversed back to its original colour within $5 \mathrm{~min}$ (as soon as they became dry).

The visible change in feather colour once wet could be attributed either to water infiltrating the air spaces in the melanin rods or to keratin swelling after absorbing water, or a combination of both. An investigation on the colour change on the scales of longhorn beetles when wet concluded by determining the structure as multilayer, with two alternating layers. ${ }^{39}$ The first layer is a melanoprotein layer, whilst the second is a layer containing melanoprotein nanoparticles and air voids. The colour change was due to the absorption of water by the first melanoprotein layer and the infiltration of water into the air voids of the second layer. We suggest that a similar process was plausible in our case, where the water was absorbed by the keratin in the surface layer and the water also penetrated the air voids in the hollow melanin granules within the multilayer. Consequently, the refractive index contrast at the interfaces between air and keratin/melanin reduced, resulting in the constructive interference of light at a modified wavelength. Notice that about half of the amino acids in the feather keratin structure are hydrophilic and half are hydrophobic. ${ }^{40}$ The surface properties of the fibre are mainly dependent on the number of hydrophobic amino acids that are in the core or external to the surface of the fibre. The longhorn beetle responded to the wet state with a change in colour in a few minutes, ${ }^{39}$ whereas the Himalayan monal feather responded in a few seconds because of the porosity in the iridescent distal part of the feather, increasing the surface area available for keratin to absorb the water. Also, the capillary effect due to the pores that quickly incorporated water in the nano/microvoids within the feathers' body cannot be ruled out.

\section{Refractive index based sensing and possible surface tension effects}

Different solutions (alcohols: glycerol and ethanol and glucose: 20 and $200 \mathrm{mM}$ solutions) were used to soak red, green and blue feathers to observe the subsequent colour shifts. The colorimetric measurements were recorded via optical microscopy and by taking the spectral data for all immersion solutions (Fig. 7). Ethanol gave a pink appearance to the red feather barbules, glycerol showed a purple colour, whereas 20 and $200 \mathrm{mM}$ glucose solutions made the feather dull and less reflective with a peak shift to $495 \mathrm{~nm}$, showing a blue appearance. The spectral measurements of the green and blue feathers showed similar trends (Fig. 6), with the major difference in colour observed with the glucose and water liquids compared to the alcohols and dry state.

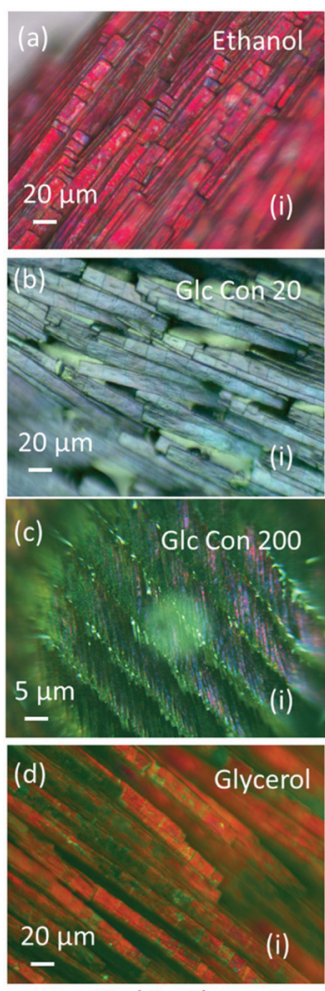

Red Feather

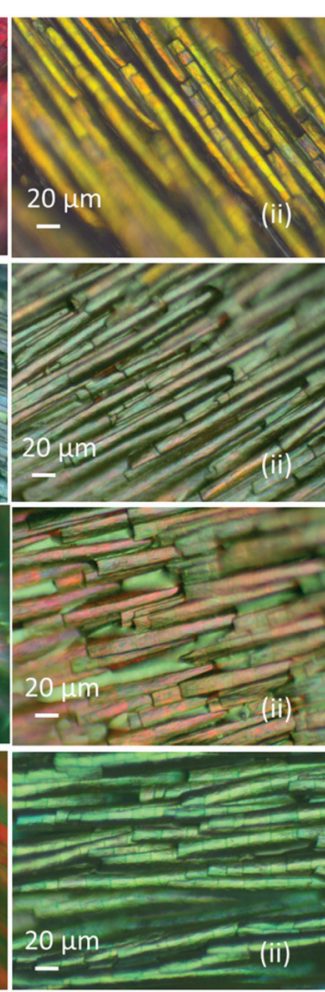

Green Feather
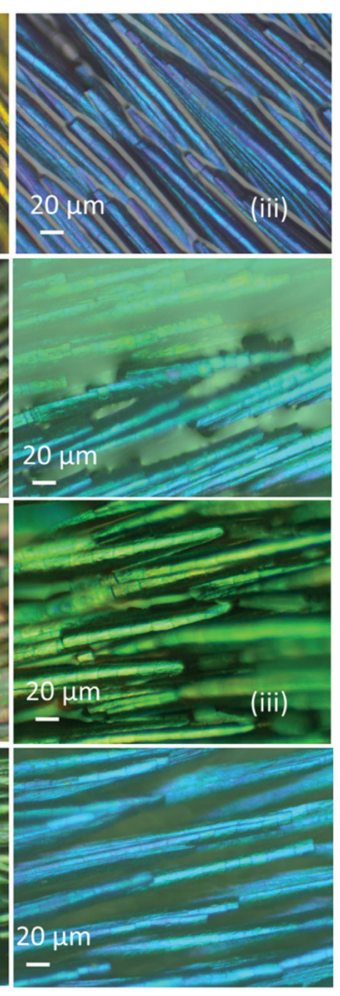

Blue Feather
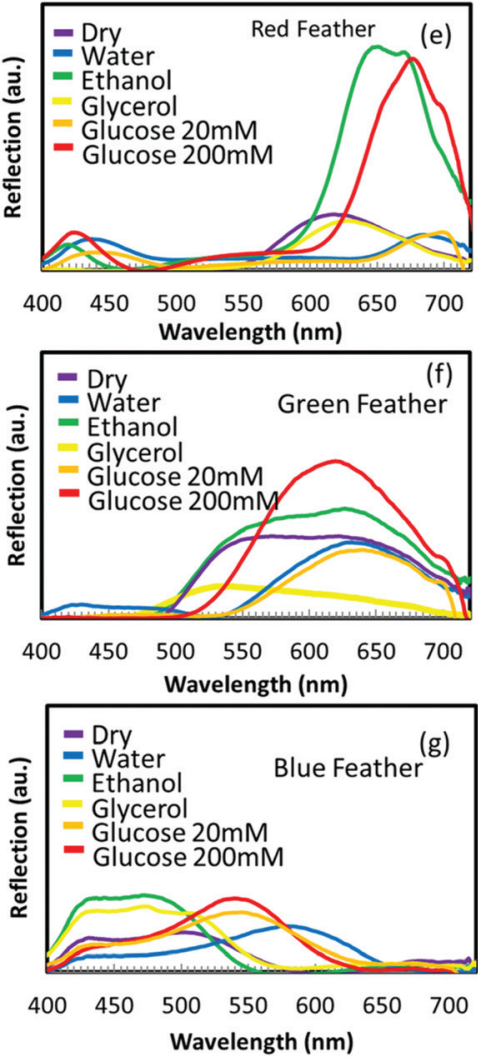

Fig. 7 Sensing of different solutions. (a-d) Microscopic images of red (i), green (ii) and blue (iii) feathers immersed in (a) ethanol, (b) $20 \mathrm{mM}$ glucose solution (c) $200 \mathrm{mM}$ glucose solution and (d) glycerol. (e-g) Reflection spectra with various solutions added to the red, green and blue feathers, respectively. 
For all feathers, the difference in colour response of ethanol on the feather compared to glycerol was more pronounced than the slight difference between the colour response of $20 \mathrm{mM}$ and $200 \mathrm{mM}$ glucose concentration, even though the difference in the refractive index between ethanol and glycerol (0.11) is half the difference between $20 \mathrm{mM}$ and $200 \mathrm{mM}$ glucose concentration (0.22). The reason for not showing a drastic change in colour between $20 \mathrm{mM}$ and $200 \mathrm{mM}$ glucose concentration solution may possibly be due to the appreciable change in the surface tension of the glucose solution - in fact, the surface tension of the glucose solution exceeds the surface tension of pure water. However, ethanol may wet the keratin completely and cause swelling for having a much more dominant effect on the feather barbules due to the change in the physical size of the feather along with the possibility of refractive index modulation. This means that the refractive index contrasts at the interfaces of air, keratin and melanin are different for each solution having different refractive indexes and surface tension leading to different colour changes in each case. Therefore, the physical mechanism responsible for the color modulation is different when different liquids having varying surface tensions are present on the feather's surface. A high surface tension liquid such as water $\left(72.8 \mathrm{mN} \mathrm{m}^{-1} \text { @ } 20^{\circ} \mathrm{C}\right)^{41}$ can cause pure refractive index modulation as the strong network of hydrogen bonds between the water molecules are more likely to stay together instead of completely wetting the underneath feather surface and subsequently getting absorbed into the body of feathers. The addition of sugar to water only increases the surface tension. Glycerol is also a highly polar liquid and offers high surface tension $\left(64.0 \mathrm{mN} \mathrm{m}^{-1}\right.$ @ $\left.20{ }^{\circ} \mathrm{C}\right) .{ }^{41}$ In contrast to the above two liquids, ethanol has low surface tension $\left(22.5 \mathrm{mN} \mathrm{m}^{-1}\right.$ (a) $\left.20^{\circ} \mathrm{C}\right),{ }^{41}$ and it even shows the tendency to wet oleophobic surfaces - it has been shown that the surfaces which repel water and ethylene glycol might not be able to repel ethanol, suggesting excellent wetting behaviour of ethanol. ${ }^{42}$ Alcohols can also cause swelling of crystalline $\alpha$-keratin, ${ }^{43}$ which is a material feathers are made up of - the bulk of the feather is expected to get wet and swell in ethanol but which might not be the case in water. Therefore, it is not only the change in the refractive index that takes part in such color modulation but also the size modulation of feathers that causes the changes in color which can even be more dominant in the case of alcohols. We conclude that one cannot detach the interfacial effects between feathers and different liquids having different surface tensions.

\section{Conclusions}

The Himalayan monal's feathers were found to be iridescent, due to the $2 \mathrm{D}$ photonic crystals present in the body of its feathers. The TEM images of the feathers taken from different parts of the bird's body revealed an RS-type structure present in all feathers according to Durrer's classification. The bird's feathers show poor hydrophobicity in contrast with many other avian species. When exposed to the liquid, the feathers showed a rapid change in their colours that reversed within $\sim 5$ minutes upon drying. The effective refractive index and swelling of the entire (/partial) structure of the feather was responsible for such colour modulation. The property of changing colour was exploited to sense other liquids. The reflection spectra were recorded to sense alcohols and other water-based solutions, which suggested that alcohols can be sensed more effectively as they produced a huge spectral shift in the reflected colour.

\section{Methods}

Optical microscopy was carried out using a microscope (Axio Scope.A1, ZEISS, Jena, Germany). BF and DF imaging was performed and the spectra were recorded using optic fibres.

The microscopic images were captured using a camera placed on top of the microscope connected to the computer using ZEN imaging software (ZEISS, Jena, Germany). The spectra were recorded using Ocean View software (Halma PLC, Amersham), with a $2.0 \mathrm{~nm}$ resolution spectrophotometer (USB2000+, Ocean Optics, Oxford) that transmits broadband light through an optical fibre connected to the microscope.

Spectroscopic measurements were carried out on the feather samples using a spectrophotometer (USB2000+, Ocean Optics, Oxford) along with OceanView software installed in the computer. A special optic fibre having a combination of seven optic fibres inside was used to transmit and receive the reflected light from the feather. Spectra and images were captured on a spot (diameter $=4 \mathrm{~mm}$ ) on the feather surface. Photographic images of each feather were then taken using a fixed camera.

The sessile drop method was used to evaluate the contact angle between the feather's surface and the water droplet. A droplet of $2 \mu \mathrm{l}$ was placed on the proximal and distal ends of the feather using a micropipette (SP0020-Auto, SciQuip, Shropshire). The feather was rotated in order to change the tilt angle from $0^{\circ}$ and $90^{\circ}$. A high-resolution camera was used to capture the images. ImageJ (Wayne Rasband, National Institute of Health, USA) software was used for analysis and measurements. Transmission electron microscopy (HT 7700) was used to analyse the internal structure of the barbules, and scanning electron microscopy (FEI Quanta 3D FEG FIB-SEM) was used to analyse the surface structure of the barbules. The feather was coated with $\mathrm{Au}$ (thickness $10 \mathrm{~nm}$ ) using DC sputtering to prevent the charging effect during scanning electron microscopy.

The feather was first placed under the optical microscope (Axio Scope.A1, ZEISS, Jena, Germany) and the relevant area was brought into focus. Images were captured using the ZEN imaging software. A droplet of $2 \mu \mathrm{l}$ was then dropped using the micropipette on the same spot under focus. The barbules absorbed the liquid droplet. Images were recorded using ZEN imaging software for wet conditions as well as in the dry state when the feather dried. Various liquids and solutions were 
used with difference in refractive indices. Images were captured in each case. Reflection spectra received were also recorded in the same manner. An optic fibre was connected to the microscope in the place of the camera. Ocean View software and a spectrophotometer (USB2000+, Ocean Optics, Oxford) were used to receive and view the optical spectra, respectively. Droplets of different liquids and solutions were used to see the effect, and the spectra received were recorded. The results were then plotted together to observe the peak shifts in wavelengths.

\section{Conflicts of interest}

There are no conflicts to declare.

\section{Acknowledgements}

H. B. thanks Khalifa University of Science and Technology (KUST) for the Faculty Startup Project (Project code: 8474000211-FSU-2019-04) and KU-KAIST Joint Research Center (Project code: 8474000220-KKJRC-2019-Health1) research funding in support of this research.

\section{References}

1 A. R. Parker, 515 Million years of structural colour, J. Opt. A: Pure Appl. Opt., 2000, 2(6), R15.

2 V. Sharma, et al., Structural origin of circularly polarized iridescence in jeweled beetles, Science, 2009, 325(5939), 449-451.

3 P. Vukusic and D. G. Stavenga, Physical methods for investigating structural colours in biological systems, J. R. Soc., Interface, 2009, 6(Suppl 2), S133-S148.

4 H. Butt, et al., Morpho butterfly-inspired nanostructures, Adv. Opt. Mater., 2016, 4(4), 497-504.

5 H. A. Hespenheide, A novel mimicry complex: beetles and flies, J. Entomol., Ser. A: Gen. Entomol., 1973, 48(1), 49-55.

6 C. M. Eliason and M. D. Shawkey, A photonic heterostructure produces diverse iridescent colours in duck wing patches, J. R. Soc., Interface, 2012, 9(74), 2279-2289.

$7 \mathrm{~J} . \mathrm{Zi}$, et al., Coloration strategies in peacock feathers, Proc. Natl. Acad. Sci. U. S. A., 2003, 100(22), 12576-12578.

8 D. Osorio and A. D. Ham, Spectral reflectance and directional properties of structural coloration in bird plumage, J. Exp. Biol., 2002, 205(14), 2017.

9 D. G. Stavenga, et al., Kingfisher feathers - colouration by pigments, spongy nanostructures and thin films, J. Exp. Biol., 2011, 214(23), 3960.

10 J. Sun, B. Bhushan and J. Tong, Structural coloration in nature, RSC Adv., 2013, 3(35), 14862-14889.

11 S. Kinoshita and S. Yoshioka, Structural colors in nature: the role of regularity and irregularity in the structure, ChemPhysChem, 2005, 6(8), 1442-1459.
12 S. M. Doucet and M. G. Meadows, Iridescence: a functional perspective, J. R. Soc., Interface, 2009, 6(Suppl 2), S115S132.

13 R. O. Prum, The anatomy and physics of avian structural colours, 2009.

14 J. D. Joannopoulos, et al., Photonic crystals: molding the flow of light, Princeton University Press, 2011.

15 H. Durrer, et al., Biology of the integument, the skin of birds, 1986.

16 G. E. Hill and K. J. McGraw, Bird coloration: mechanisms and measurements, Harvard University Press, 2006, vol. 1.

$17 \mathrm{H}$. Yin, et al., Iridescence in the neck feathers of domestic pigeons, Phys. Rev. E: Stat., Nonlinear, Soft Matter Phys., 2006, 74(5), 051916.

18 C. M. Eliason and M. D. Shawkey, A photonic heterostructure produces diverse iridescent colours in duck wing patches, J. R. Soc., Interface, 2012, 9(74), 2279-2289.

19 C. H. Greenewalt, W. Brandt and D. D. Friel, Iridescent colors of hummingbird feathers, J. Opt. Soc. Am., 1960, 50(10), 1005-1013.

20 M. Land, The physics and biology of animal reflectors, Prog. Biophys. Mol. Biol., 1972, 24, 75-106.

21 A. K. Yetisen, et al., Light-directed writing of chemically tunable narrow-band holographic sensors, Adv. Opt. Mater., 2014, 2(3), 250-254.

22 H. Butt, Q. Dai, T. D. Wilkinson and G. A. J. Amaratunga, Photonic crystals \& metamaterial filters based on 2D arrays of silicon nanopillars, Prog. Electromagn. Res., 2011, 113, 179-194.

23 A. K. Yetisen, et al., Photonic hydrogel sensors, Biotechnol. Adv., 2016, 34(3), 250-271.

24 I. Rashid, et al., Structural coloration in caloenas nicobarica pigeons and refractive index modulated sensing, Adv. Opt. Mater., 2018, 6(9), 1701218.

25 A. K. Yetisen, et al., Photonic nanosensor for colorimetric detection of metal ions, Anal. Chem., 2015, 87(10), 51015108.

26 A. K. Yetisen, et al., Color-selective 2.5D holograms on large-area flexible substrates for sensing and multilevel security, Adv. Opt. Mater., 2016, 4(10), 1589-1600.

$27 \mathrm{Y}$. Xu, et al., Optical refractive index sensors with plasmonic and photonic structures: promising and inconvenient truth, Adv. Opt. Mater., 2019, 7(9), 1801433.

28 S. M. Doucet, et al., Iridescent plumage in satin bowerbirds: structure, mechanisms and nanostructural predictors of individual variation in colour, J. Exp. Biol., 2006, 209(2), 380-390.

29 D. G. Stavenga, et al., Dramatic colour changes in a bird of paradise caused by uniquely structured breast feather barbules, Proc. R. Soc. London, Ser. B, 2010, 278, 20982104.

$30 \mathrm{H}$. Durrer, Schillerfarben der vogelfeder als evolutionsproblem: elektronenmikroskopische untersuchung der schillerstrukturen, ihrer morphogenese und analyse von selektionsmechanismen (speziell dargelegt am beispiel der hühnervögel), Fretz, 1977. 
31 Q. Zhao, et al., Bio-inspired sensing and actuating materials, J. Mater. Chem. C, 2019, 7(22), 64936511.

32 H. Zhou, et al., Bio-Inspired Photonic Materials: Prototypes and structural effect designs for applications in solar energy manipulation, Adv. Funct. Mater., 2018, 28(24), 1705309.

33 D. G. Stavenga, et al., High refractive index of melanin in shiny occipital feathers of a bird of paradise, Light: Sci. Appl., 2015, 4, e243.

34 T. Zhu, et al., A transparent superhydrophobic coating with mechanochemical robustness for anti-icing, photocatalysis and self-cleaning, Chem. Eng. J., 2020, 399, 125746.

35 A. Marmur, Hydro- hygro- oleo- omni-phobic? Terminology of wettability classification, Soft Matter, 2012, 8(26), 68676870.

36 C. Eliason and M. Shawkey, Decreased hydrophobicity of iridescent feathers: a potential cost of shiny plumage, J. Exp. Biol., 2011, 214(Pt 13), 2157.
37 X. Su, et al., Bioinspired superhydrophobic thermochromic films with robust healability, ACS Appl. Mater. Interfaces, 2020, 12(12), 14578-14587.

38 B. A. Walther and D. H. Clayton, Elaborate ornaments are costly to maintain: evidence for high maintenance handicaps, Behav. Ecol., 2004, 16(1), 89-95.

39 F. Liu, et al., Structural color change in longhorn beetles Tmesisternus isabellae, Opt. Express, 2009, 17(18), 1618316191.

40 W. F. Schmidt and S. Jayasundera, Microcrystalline avian keratin protein fibers, in Natural Fibers, Plastics and Composites, Springer, 2004, pp. 51-66.

41 J. Yong, et al., Superoleophobic surfaces, Chem. Soc. Rev., 2017, 46, 4168-4217.

42 T. Darmanin and F. Guittard, Superhydrophobic and superoleophobic properties in nature, Mater. Today, 2015, 18(15), 273-285.

43 M. Feughelman and J. W. Snaith, The swelling of crystalline $\alpha$-keratin by alcohols, Biochim. Biophys. Acta, Spec. Sect. Biophys. Subj., 1964, 79(1), 203-205. 